\title{
A Routing Calculus with Distance Vector Routing Updates
}

\author{
Priyanka Gupta, Manish Gaur \\ Centre For Advanced Studies \\ Dr. A.P.J. Abdul Kalam Technical University \\ Lucknow, India
}

\begin{abstract}
We propose a routing calculus in a process algebraic framework to implement dynamic updates of routing table using distance vector routing. This calculus is an extension of an existing routing calculus $D_{\pi}^{\omega}$ where routing tables are fixed except when new nodes are created in which case the routing tables are appended with relevant entries. The main objective of implementing dynamic routing updates is to demonstrate the formal modeling of distributed networks which is closer to the networks in practice. We justify our calculus by showing its reduction equivalence with its specification $D_{\pi}$ (distributed $\pi$ calculus) after abstracting away the unnecessary details from our calculus which in fact is one of the implementations of $D_{\pi}$. We nomenclate our calculus with routing table updates as $\mathbf{D R}_{\pi}^{\varphi}$.
\end{abstract}

Keywords-Routing Calculi; Routing Protocols; Well Formed Configuration; Reduction Semantics

\section{INTRODUCTION}

In recent years, developments in formal modeling of distributed networks in a process algebraic framework through process calculi has marked profound work [1], [2] [3], [4], [5], [6], [7], [8], [9], [10], [11], [12]. The extended version of Asynchronous Distributed $\pi$-calculus $\left(\mathrm{AD}_{\pi}\right)$ named as routing calculi $\mathrm{DR}_{\pi}^{\omega}$ was one of the significant developments towards modeling the distributed computer network using an active component named router and considering the path of a communication between the communicating nodes where a routing table is a dynamic entity in a typical distributed network.

$\mathrm{DR}_{\pi}^{\omega}$ consists of a network of routers of fixed topology. The processes reside in a located site called nodes which are directly connected to some specific router. Any two processes at nodes can communicate through the routers. The routers find the path over the network between the communicating processes. The processes communicate via this path.

A system in $\mathrm{DR}_{\pi}^{\omega}$, looks like $\Gamma_{c} \triangleright S$. Here $S$ can be of the form $\langle R\rangle \llbracket n[P] \rrbracket$ where $P$ is a process that resides under node $n$ connected at the same or a different node to some router $R$. The communication between the processes takes place through routers. Each process is located at some particular node which in turn resides at some particular router. The routers determine the particular path along the router connectivity through which the communicated values are forwarded. In this language, the routing table is updated only when a new node is created which limits dynamic updates of the table.

We present a new calculi $\mathrm{DR}_{\pi}^{\varphi}$ which is a direct adoption of routing calculus $\mathrm{DR}_{\pi}^{\omega}$ [10] with a modified feature of routing table updates which is dynamic in $\mathrm{DR}_{\pi}^{\varphi}$ unlike $\mathrm{DR}_{\pi}^{\omega}$. We have

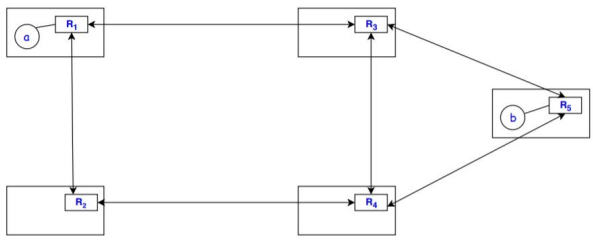

Fig. 1. A simple distributed with routers and nodes

abstracted away few details from $\mathrm{DR}_{\pi}^{\omega}$ to demonstrate the power of new calculi in more simple way. These features can be adopted to $\mathrm{DR}_{\pi}^{\varphi}$ without much amendments to it. We describe a method for routing table updates with the help of implementation of distance vector routing method [13], [14], [15], [16] which uses the Bellman-Ford algorithm [17], [18], [19], [20] to compute the shortest route. In this calculi, the routing tables are periodically exchanged with their neighbors and with this updated entries new routes are found. We abstract away the details of new routes calculation methods by incorporating function $\delta$ in our semantics rule. Further to maintain the consistency in the calculi a clock $t_{k^{\prime}}$ is introduced so that the routing table exchange and thereafter update calculation are done at discrete time. The condition in well formed configuration ensure that the calculi remains consistent in term of self looping of message propagation, path guarantee etc. This calculi presents more realistic picture of distributed networks with routers and therefore is closer to the real implementation.

In $\mathrm{DR}_{\pi}^{\varphi}$, a system is represented by $\left\langle R^{t_{k^{\prime}}}\right\rangle \llbracket n[P] \rrbracket$ where a process $P$ is located at node $n$. The node $n$ is directly connected to the router $R$ at global clock $t=t_{k^{\prime}}$. The system is accompanied with the router connectivity $\Gamma_{c}$. Hence, the configuration paves the way to reductions. A configuration $\Gamma_{c} \triangleright S$ comprises of router connectivity $\Gamma_{c}$ and system $S$.

In the following sections the paper is organized as follows:

The syntax , structural equivalence and reduction semantics of $\mathrm{DR}_{\pi}^{\varphi}$ are described in Section 2, 3 and 4 respectively. We have explained an example to illustrate the reduction rules more clearly in Section 5. We require certain conditions on well formed configuration for the consistent behavior of the reduction semantics in Section 6. We describe the equivalence between $\mathrm{DR}_{\pi}^{\varphi}$ and $\mathrm{D}_{\pi}$ in Section 7. The conclusion is in Section 8 . 


\section{SYNTAX}

We will use $v, v_{1}, v_{2}, u, u_{1} \ldots$ to represents values which may be a simple value or a name or a variable. For simplicity in the language we don't use tuples as values. Therefore $u, v, \ldots$. are singleton names or simple values i.e. integers, boolean etc. We use meta variables $a, b, c, \ldots$ to range over channel names $\complement$ or node names $N$. In the description of the language $n, m, \ldots$ are used to range over node names $N$ and we use $R, R_{1}, R_{2}, \ldots$ to range over set of router names $\Re$ at global time $t_{k}, t_{k+1}, t_{k+2}, \ldots$ . The variables $h, l, \ldots$ range over integers to represent the cost of communication.

Further, we assume that sets of node names, router names and channel names are disjoint from each other. More formally

$$
\Re \cap \complement \cap N=\Phi
$$

There exists three main syntactic categories in the language that are Nodes, Systems, and Processes. We described the syntax of $\mathrm{DR}_{\pi}^{\varphi}$ in Fig. 1. We have given the descriptions of these syntactic categories in the following sub-sections.

\section{A. System}

In Fig. 2, we described a system as $\left\langle R^{t_{k^{\prime}}}\right\rangle \llbracket M \rrbracket$ where $R$ being a router at global clock $t_{k^{\prime}}$ and $M$ is a another syntactic category named as nodes that are directly connected to $R$. $S \mid T$ represents two parallel systems and $[R] M_{s g}^{h}(n, m, v @ c)$ is a message at router $R$. This message is used to propagate the value $v$ from one router to another during communication between some process at source node $n$ to another process at destination node $m$. The value propagated by the message is represented by $v @ c$ to deliver value $v$ to the specified channel $c$ of the destination process. Here the integer $h$ indicates the number of hops(routers), the message has already travelled across the path towards its destination and $\varepsilon$ is the identity.

\section{B. Node}

In Fig. 2, the nodes are named processes $n[P]$ where $n$ is the name of a node and $P$ is a process term in it. $M \mid N$ describes usual concurrency between nodes $M$ and $N$ at any router. As an example, in a system $\left\langle R^{t_{k^{\prime}}}\right\rangle \llbracket M \mid N \rrbracket$ the nodes $M$ and $N$ are running in parallel at router $R$ at global clock $t_{k^{\prime}} .0$ is the identity.

\section{Process terms}

The process terms are very similar to the terms in [1], [5]. These process terms are described in Fig. 2.

\section{Structural EQUivalence}

We introduce a formal relation between the system terms in $\mathrm{DR}_{\pi}^{\varphi}$ called structural equivalence which is represented by the notation $\equiv$ to this relation, they are same computational entity. This is defined in [1], [5]. We describe the definition of structural equivalence is separated for all syntactic categories. Nevertheless, the node equivalence inherits process equivalence and system equivalences inherits by node equivalence. For example, the terms $\left\langle R^{t_{k^{\prime}}}\right\rangle \llbracket M_{1} \mid M_{2} \rrbracket$ and $\left\langle R^{t}{k^{\prime}}^{\prime}\right\rangle \llbracket M_{2} \mid M_{1} \rrbracket$, instinctively represent the same systems where the nodes $M_{1}$ and $M_{2}$ at router $R$ run in parallel at global clock $t_{k^{\prime}}$ and the

$\begin{array}{ll}S, T::= & \text { Systems } \\ \left\langle R^{t_{k^{\prime}}}\right\rangle \llbracket M \rrbracket & \text { Router at globa } \\ S \mid T & \text { Concurrency } \\ {[R] M_{s g}^{h}(n, m, v @ c)} & \begin{array}{l}\text { Messages } \\ \varepsilon\end{array} \\ & \text { Identity } \\ M, N::= & \text { Nodes } \\ n[P] & \\ M \mid N & \text { Named proces } \\ 0 & \text { Concurrency } \\ T, U::= & \text { Identity } \\ c ?(x) P & \text { Process Term } \\ m !(v @ c) & \\ \text { if } v_{1}=v_{2} \text { then } P \text { else } Q & \begin{array}{l}\text { Matching } \\ P \mid Q\end{array} \\ * P & \text { Concurrency } \\ \text { stop } & \text { Recursive } \\ & \text { Termination }\end{array}$

Fig. 2. Syntax $\mathrm{Of} \mathrm{DR}_{\pi}^{\varphi}$

$\begin{array}{lc}\text { (SE-COM) } & P|Q \equiv Q| P \\ \text { (SE-ASSOC) } & (P \mid Q)|R \equiv P|(Q \mid R) \\ \text { (SE-ID) } & P \mid i d \equiv P\end{array}$

Fig. 3. Structural Equivalence(Standard) for $\mathrm{DR}_{\pi}^{\varphi}$

(SE-P-STANDARD) standard axioms

(SE-P-Recursion) $\quad * P \equiv P \mid * P$

Fig. 4. Structural Equivalence(Processes) for $\mathrm{DR}_{\pi}^{\varphi}$

$\begin{array}{lr}\text { (SE-N-STANDARD) } & \text { standard axioms } \\ \text { (SE-N-STOP) } & m[\text { stop }] \equiv 0 \\ \text { (SE-N-INHERITANCE) } & \equiv Q \equiv Q \\ & m[P] \equiv m[P]\end{array}$

Fig. 5. Structural Equivalence(Nodes) $\mathrm{DR}_{\pi}^{\varphi}$

\begin{tabular}{ll} 
(SE-S-STANDARD) & standard axioms \\
(SE-S-INHERITANCE) & $N \equiv S$ \\
\cline { 2 - 3 } & $\left\langle R^{t_{k^{\prime}}}\right\rangle \llbracket N \rrbracket \equiv\left\langle R^{t_{k^{\prime}}}\right\rangle \llbracket S \rrbracket$
\end{tabular}

Fig. 6. Structural Equivalence(Systems) for $\mathrm{DR}_{\pi}^{\varphi}$

order of their composition really does not matter. These are defined in Fig. 2, 3, 4, 5 and 6.

\section{Reduction SEMANTiCS}

The reduction semantics are defined on configurations $\Gamma_{c} \triangleright S$. The configuration reduction step is defined as $\Gamma_{c} \triangleright$ $S \longrightarrow{ }^{h} \Gamma_{c} \triangleright S^{\prime}$ where the cost of reduction [3], [4] is $h$ and a system $S$ reduces $S^{\prime}$. These reduction rules for $\mathrm{DR}_{\pi}^{\varphi}$ are given in Fig. 7 and directly inherited from [8], [10]. The Rule (ROUT) is for delivery. For example, let us take the configuration $\Gamma_{c} \triangleright\left\langle R^{t_{k^{\prime}}}\right\rangle \llbracket n[m !(v @ c)$

$\mid P] \mid N \rrbracket$ where a process $m !(v @ c) \mid P$ at source node $n$ at router $R$ at a global clock $t_{k^{\prime}}$ outputs a value $v$ at channel $c$ which is located at some process at destination node $m$. This reduction rule generates a propagation message $[R] M_{s g}^{0}(n, m, v @ c)$ in parallel with the system $\left\langle R^{t_{k^{\prime}}}\right\rangle \llbracket n[P] \mid N \rrbracket$ resulting in a configu- 
ration $\quad \Gamma_{c} \triangleright[R] M_{s g}^{0}(n, m, v @ c)\left|\left\langle R^{t_{k^{\prime}}}\right\rangle \llbracket n[P]\right| N \rrbracket$. The message with subscript 0 indicates that it has been generated at router $R$ and has not hopped to any other router yet. The term $(n, m, v @ c)$ in the message represents the source node name $n$ where a process outputs a value and destination node $m$ where the value $v$ is to be delivered on channel $c$ to a waiting process.

The propagation of the message from one router to another router towards the destination node is done using reduction rule (R-MSG-FWD) in Fig. 7 . Let us consider the reduction rule (R-MSG-FWD). In this reduction rule a configuration of the form $\Gamma_{c} \triangleright\left[R_{1}\right] M_{s g}^{h}(n, m, v @ c)\left|\left\langle R_{2}{ }^{t_{k^{\prime}}}\right\rangle \llbracket N \rrbracket\right| S$ reduced to $\Gamma_{c} \triangleright$ $\left[R_{2}\right] M_{s g}^{h+1}(n, m, v @ c)\left|\left\langle R_{2}{ }^{t_{k^{\prime}}}\right\rangle \llbracket N \rrbracket\right| S$. There are two premises the first $\left(R_{1}, R_{2}\right) \in \Gamma_{c}$ means that the routers $R_{1}$ and $R_{2}$ are directly connected or $R_{2}$ is a neighbor of $R_{1}$. The second one, $\left\langle R_{1}{ }^{t}{ }_{k^{\prime}}\right\rangle(m)=R_{2}$ means that $\mathrm{m}$ belongs to the domain of the routing table at $R_{1}$ at global clock $t_{k^{\prime}}$ and the function $\left\langle R_{1}{ }^{{ }^{\prime}}\right\rangle$ returns $R_{2}$ as the next hop towards the destination node $m$.

In the reduction rule (R-COMM) in Fig. 7, a configuration $\quad \Gamma_{c} \triangleright[R] M_{s g}^{h}(n, m, v @ c)\left|\left\langle R^{t_{k^{\prime}}}\right\rangle \llbracket m[c ?(x) P \mid Q]\right| N \rrbracket$ does a reduction to $\Gamma_{c} \triangleright\left\langle R^{t} k^{\prime}\right\rangle \llbracket m[P\{v / x\} \mid Q] \mid N \rrbracket$. Here note that cost of reduction is $h$ as the message has hopped $h$ routers from source node $n$ to destination node $m$. However it is not necessary that for every hop the global clock count increases by an interval.

we describe the reduction rule (R-TABLE-UPDATE) in Fig. 7 which uses a new special notation $(\longleftrightarrow)$ to depict the exchange of routing tables at global clock $t=t_{k^{\prime}}$. This notion $(\longleftrightarrow)$ adds a novelty to this calculus as this will not only exchange the routing table between the connecting routers but also update tables (using distance vector routing methods [13], [14], [15], [16] dynamically with the help of synchronization of the global clock. Here we define reduction semantics for updating table dynamically, may or may not at global clock $t_{k^{\prime}}$. In this reduction rule a configuration of the form $\left\langle R_{1}{ }^{t_{k^{\prime}+1}}\right\rangle[M] \mid S$ reduces to $\left\langle R_{1}^{\prime t_{k^{\prime}+1}}\right\rangle[M] \mid S$. There are six premises, the first $\left(R_{1}, R_{2}\right) \in \Gamma_{c}$ means that the routers $R_{1}$ and $R_{2}$ are directly connected or $R_{2}$ is a neighbor of $R_{1}$ at global clock $t=t_{k^{\prime}}$. The second and third, $\Gamma_{c} \triangleright$ $\left\langle R_{1}{ }^{t}{ }_{k^{\prime}}\right\rangle[M] \mid S$ and $\left\langle R_{2}{ }^{t_{k^{\prime}}}\right\rangle[N] \mid T$ are well formed which mean that the well formedness is preserved under reductions. The fourth, $\left\langle R_{1}{ }^{t}{ }_{k^{\prime}}\right\rangle[M]\left|S \longleftrightarrow\left\langle R_{2}{ }^{{ }^{k^{\prime}}}\right\rangle[N]\right| T$ means that the routing table between the connecting routers is exchanged. The fifth and sixth, $\delta\left\langle R_{1}{ }^{t}{ }_{k^{\prime}+1}\right\rangle=\left\langle R_{1}^{\prime t^{\prime}+1}\right\rangle$ and $\delta\left\langle R_{2}{ }^{t_{k^{\prime}+1}}\right\rangle=\left\langle R_{2}^{\prime}{ }_{k^{\prime}+1}\right\rangle$ mean that routing tables (using distance vector routing methods) are updated dynamically with the help of synchronization of the global clock $t_{k^{\prime}}$.

The rules (R-MATCH) and (R-MISMATCH) are tests for values. Here the initial cost of these reductions is also zero. The compositional rules are defined in the rule (R-CONTX) in Fig. 7 and are preserved under the static operator $\mid$. The other reduction rule (R-STRUCT) in Fig. 7 defines well formed configuration reduction upto system structural equivalence.

Now we will demonstrate these rules with the help of an example. This example also shows the exclusive feature of this particular language regarding the novel rule is implemented for routing table updates.

\section{EXAMPLE}

In Fig. 1, let us assume that a system $S$ is defined as $S_{1}\left|S_{2}\right| S_{3}\left|S_{4}\right| S_{5}$ where
(R-OUT)

$\Gamma_{c} \triangleright\left\langle R^{t_{k^{\prime}}}\right\rangle \llbracket n[m !(v @ c) \mid P] \mid N \rrbracket \rightarrow$

$\Gamma_{c} \triangleright[R] M_{s g}^{0}(n, m, v @ c)\left|\left\langle R^{t_{k^{\prime}}}\right\rangle \llbracket n[P]\right| N \rrbracket$

(R-COMM)

$\frac{\left\langle R^{t_{k^{\prime}}}\right\rangle(m)=R}{\Gamma_{c} \triangleright[R] M_{s g}^{h}(n, m, v @ c)\left|\left\langle R^{t_{k^{\prime}}}\right\rangle \llbracket m[c ?(x) P \mid Q]\right| N \rrbracket \longrightarrow}$
$\Gamma_{c} \triangleright\left\langle R^{t_{k^{\prime}}}\right\rangle \llbracket m[P\{v / x\} \mid Q] \mid N \rrbracket$

(R-MSG-FWD)

$$
\begin{gathered}
\left(R_{1}, R_{2}\right) \in \Gamma_{c} \\
\left\langle R_{1} t_{k^{\prime}}\right\rangle(m)=R_{2} \\
\hline \Gamma_{c} \triangleright\left[R_{1}\right] M_{s g}^{h}(n, m, v @ c) \mid\left\langle R_{2} t_{k^{\prime}} \llbracket N \rrbracket\right| S \longrightarrow \\
\Gamma_{c} \triangleright\left[R_{2}\right] M_{s g}^{h+1}(n, m, v @ c) \mid\left\langle R_{2}{ }^{t_{k^{\prime}}} \llbracket N \rrbracket\right| S
\end{gathered}
$$

(R-MATCH)

$\Gamma_{c} \triangleright\left\langle R^{t} k_{k^{\prime}}\right\rangle \llbracket n[$ if $v=v$ then $P$ else $Q] \rrbracket \longrightarrow \Gamma_{c} \triangleright\left\langle R^{t} k^{\prime}\right\rangle \llbracket n[P] \rrbracket$

(R-MISMATCH)

$\Gamma_{c} \triangleright\left\langle R^{t_{k^{\prime}}}\right\rangle \llbracket n\left[\right.$ if $v_{1} \neq v_{2}$ then $P$ else $\left.Q\right] \rrbracket \longrightarrow \Gamma_{c} \triangleright\left\langle R^{t_{k^{\prime}}}\right\rangle \llbracket n[Q] \rrbracket$

\section{(R-TABLE-UPDATE)}

$$
\begin{gathered}
\left(R_{1}, R_{2}\right) \in \Gamma_{c}, t=t_{k^{\prime}} \\
\Gamma_{c} \triangleright\left\langle R_{1}{ }_{k_{k^{\prime}}}\right\rangle[M] \mid S \text { is wff } \\
\Gamma_{c} \triangleright\left\langle R_{2} t_{k^{\prime}}\right\rangle[N] \mid T \text { is wff } \\
\left\langle R_{1}{ }^{t}{ }_{k^{\prime}}\right\rangle[M]\left|S \longleftrightarrow\left\langle R_{2}{ }^{t_{k^{\prime}}}\right\rangle[N]\right| T \\
\delta\left\langle R_{1}{ }_{k^{\prime}+1}\right\rangle=\left\langle R_{1}^{\prime}{ }_{k^{\prime}+1}\right\rangle \\
\delta\left\langle R_{2}{ }^{t_{k^{\prime}+1}}\right\rangle=\left\langle R_{2}^{\prime}{ }_{k^{\prime}+1}\right\rangle \\
\Gamma_{c} \triangleright\left\langle R_{1}{ }^{t}{ }_{k^{\prime}+1}\right\rangle[M]\left|S \longrightarrow \Gamma_{c} \triangleright\left\langle R_{1}^{\prime}{ }_{k^{\prime}+1}\right\rangle[M]\right| S \\
\Gamma_{c} \triangleright\left\langle R_{2}{ }^{t}{ }^{\prime}+1\right\rangle[N]\left|T \longrightarrow \Gamma_{c} \triangleright\left\langle R_{2}^{\prime}{ }_{k^{\prime}+1}\right\rangle[N]\right| T
\end{gathered}
$$

(R-STRUCT)

$$
\frac{S \equiv S^{\prime}, \Gamma_{c} \triangleright S^{\prime} \longrightarrow \Gamma_{c} \triangleright R^{\prime}, R^{\prime} \equiv R}{\Gamma_{c} \triangleright S \longrightarrow \Gamma_{c} \triangleright R}
$$

\section{(R-CONTX)}

$$
\begin{gathered}
\Gamma_{c} \triangleright S_{1} \longrightarrow \Gamma_{c} \triangleright S_{1}^{\prime} \\
\Gamma_{c} \triangleright S_{1}\left|S_{2} \longrightarrow \Gamma_{c} \triangleright S_{1}^{\prime}\right| S_{2} \\
\Gamma_{c} \triangleright S_{2}\left|S_{1} \longrightarrow \Gamma_{c} \triangleright S_{2}\right| S_{1}^{\prime}
\end{gathered}
$$

Fig. 7. Reduction Semantics for $\mathrm{DR}_{\pi}^{\varphi}$

$$
\begin{gathered}
S_{1} \equiv\left\langle R_{1}^{t_{k}}\right\rangle \llbracket P \mid N_{1} \rrbracket \\
S_{1} \equiv\left\langle R_{2}^{t_{k}}\right\rangle \llbracket \llbracket N_{2} \rrbracket \\
S_{3} \equiv\left\langle R_{3}^{t_{k}}\right\rangle \llbracket \llbracket N_{3} \rrbracket \\
S_{4} \equiv\left\langle R_{4}^{t_{k}}\right\rangle \llbracket N_{4} \rrbracket \\
S_{5} \equiv\left\langle R_{5}^{t_{k}}\right\rangle \llbracket Q \mid N_{5} \rrbracket
\end{gathered}
$$

Where $P \equiv a[b !\langle v @ c\rangle]$ and $Q \equiv b[c ?(x) R]$ The router connectivity $\Gamma_{c}$ is defined as $\left\{\left(R_{1}, R_{2}\right),\left(R_{1}, R_{3}\right)\right.$, $\left.\left(R_{2}, R_{4}\right),\left(R_{3}, R_{4}\right),\left(R_{3}, R_{5}\right),\left(R_{5}, R_{4}\right)\right\}$.

The configuration $\Gamma_{c} \triangleright S_{1}\left|S_{2}\right| S_{3}\left|S_{4}\right| S_{5}$ does a reduction using the rule (R-OUT) where the process $b !\langle v @ c\rangle$ at node $a$ generates a message at global clock $t_{k^{\prime}}$ where $t_{k^{\prime}}=$ $t_{k}, t_{k+1}, t_{k+2}, \ldots$. The configuration reduces to another configuration of the form 


$$
\Gamma_{c} \triangleright\left[R_{1}\right] M_{s g}^{0}(a, b, v @ c)\left|\left\langle R_{1}^{t_{k}}\right\rangle \llbracket N_{1} \rrbracket\right| S_{2}\left|S_{3}\right| S_{4} \mid S_{5}
$$

In the Fig. $1, R_{1}$ is directly connected to $R_{2}$ and $R_{3}$. we know that $\left(R_{1}, R_{2}\right) \in \Gamma_{c}$ and $\left(R_{1}, R_{3}\right) \in \Gamma_{c}$. Similarly we know that $\left(R_{2}, R_{4}\right) \in \Gamma_{c},\left(R_{3}, R_{4}\right) \in \Gamma_{c},\left(R_{4}, R_{5}\right) \in \Gamma_{c},\left(R_{5}, R_{3}\right) \in \Gamma_{c}$.

All the routing table will share its routing table with adjacent router. Now by using rule(R-TABLE-UPDATE), we get

$$
\begin{aligned}
& \Gamma_{c} \triangleright\left\langle R_{1}^{t_{k}}\right\rangle \llbracket|P| N_{1} \rrbracket \text { is wff } \\
& \Gamma_{c} \triangleright\left\langle R_{2}^{t_{k}}\right\rangle \llbracket N_{2} \rrbracket \text { is wff } \\
& \Gamma_{c} \triangleright\left\langle R_{3}^{t_{k}}\right\rangle \llbracket N_{3} \rrbracket \text { is wff } \\
& \Gamma_{c} \triangleright\left\langle R_{4}^{t_{k}}\right\rangle \llbracket N_{4} \rrbracket \text { is wff } \\
& \Gamma_{c} \triangleright\left\langle R_{5}^{t_{5}}\right\rangle \llbracket Q \mid N_{5} \rrbracket \text { is wff }
\end{aligned}
$$

All the systems are well formed which are defined in definition 1. Now all the routing tables shall be exchanged with each other at a global clock $t_{k}$.

$$
\begin{array}{r}
\left\langle R_{1}^{t_{k}}\right\rangle \llbracket P \mid N_{1} \rrbracket \longleftrightarrow\left\langle R_{2}^{t_{k}}\right\rangle \llbracket N_{2} \rrbracket \\
\left\langle R_{1}^{k_{k}}\right\rangle \llbracket P \mid N_{1} \rrbracket \longleftrightarrow\left\langle R_{3}^{k_{k}}\right\rangle \llbracket N_{3} \rrbracket \\
\left\langle R_{t_{k}}^{t_{k}}\right\rangle \llbracket N_{2} \rrbracket \longleftrightarrow\left\langle R_{4}^{t_{k}}\right\rangle \llbracket N_{4} \rrbracket \\
\left\langle R_{3}^{t_{k}}\right\rangle \llbracket N_{3} \rrbracket \longleftrightarrow\left\langle R_{4}^{k_{k}}\right\rangle \llbracket \llbracket N_{4} \rrbracket \\
\left\langle R_{k}^{t_{k}}\right\rangle \llbracket N_{3} \rrbracket \longleftrightarrow\left\langle R_{t_{k}}^{t_{k}}\right\rangle \llbracket Q \mid N_{5} \rrbracket \\
\left\langle R_{4}^{t_{k}}\right\rangle \llbracket N_{4} \rrbracket \longleftrightarrow\left\langle R_{5}^{k_{k}}\right\rangle \llbracket Q \mid N_{5} \rrbracket \\
\left\langle R_{5}^{t_{k}}\right\rangle \llbracket Q \mid N_{5} \rrbracket \longleftrightarrow\left\langle R_{3}^{t_{k}}\right\rangle \llbracket N_{3} \rrbracket
\end{array}
$$

Now route checks update for new information then routers will be calculated using Bellman-Ford algorithm and metric is updated, new entries are stored in the routing table. Thus routers will exchange routing information at $t_{k+1}$.

$$
\begin{aligned}
& \boldsymbol{\delta}\left\langle R_{1}{ }^{t_{k+1}}\right\rangle=\left\langle R_{1}{ }^{t_{k+1}}\right\rangle \\
& \boldsymbol{\delta}\left\langle R_{2}{ }_{k+1}\right\rangle=\left\langle R_{2}^{\prime t_{k+1}}\right\rangle \\
& \boldsymbol{\delta}\left\langle R_{3}{ }^{t_{k+1}}\right\rangle=\left\langle R_{3}^{\prime t_{k+1}}\right\rangle \\
& \boldsymbol{\delta}\left\langle R_{4}{ }_{k+1}\right\rangle=\left\langle R_{4}{ }_{k+1}\right\rangle \\
& \boldsymbol{\delta}\left\langle R_{5}{ }^{t_{k+1}}\right\rangle=\left\langle R_{5}^{\prime t_{k+1}}\right\rangle
\end{aligned}
$$

In this way $\left\langle R_{2}{ }^{t_{k+1}}\right\rangle,\left\langle R_{3}{ }^{t_{k+1}}\right\rangle$ and $\left\langle R_{5}{ }^{t_{k+1}}\right\rangle$ are updated and new routing tables are $\left\langle R_{2}^{\prime} t_{k+1}\right\rangle,\left\langle R_{3}^{\prime} t_{k+1}\right\rangle$ and $\left\langle R_{5}{ }^{\prime}{ }_{k+1}\right\rangle$ respectively at global clock $t_{k+1}$.

Now the message hops towards the destination node $b$, the router table $\left\langle R_{1}{ }^{t_{k+1}}\right\rangle$ may return either the adjacent router $R_{2}$ or the adjacent router $R_{3}$ as next hop on the communication path to node $b$ at router $R_{5}$. This may be formally expressed as $\left\langle R_{1}^{t_{k+1}}\right\rangle(b)=R_{2}$ and $\left\langle R_{1}{ }_{k+1}\right\rangle(b)=R_{3}$.

The communication path is chosen by distance vector approach (shortest path). Suppose the routing table $\left\langle R_{1}{ }^{t_{k+1}}\right\rangle$ returns $R_{3}$ as the next hop for reaching $b$. This essentially means that $R_{3}$ is on the path towards $b$ which is hosted at router $R_{5}$. Formally $\left\langle R_{1}{ }^{t_{k+1}}\right\rangle(b)=R_{3}$ and also we know that $\left(R_{1}, R_{3}\right) \in \Gamma_{c}$. Therefore with an application of rule (R-MSGFWD) the message $\left[R_{1}\right] M_{s g}^{0}(a, b, v @ c)$ hops at $R_{3}$. So the configuration

$$
\begin{gathered}
\Gamma_{c} \triangleright\left[R_{1}\right] M_{s g}^{0}(a, b, v @ c)\left|\left\langle R_{1}^{t_{k+1}}\right\rangle \llbracket P\right| N_{1} \rrbracket\left|\left\langle R_{2}^{\prime t_{k+1}}\right\rangle \llbracket \llbracket N_{2} \rrbracket\right| \\
\left\langle R_{3}^{t_{k+1}}\right\rangle \llbracket N_{3} \rrbracket\left|\left\langle R_{4}{ }^{t_{k+1}}\right\rangle \llbracket N_{4} \rrbracket\right|\left\langle R_{5}^{\prime}{ }^{\prime}{ }_{k+1}\right\rangle \llbracket Q \mid N_{5} \rrbracket
\end{gathered}
$$

reduces to

$$
\begin{aligned}
& \Gamma_{c} \triangleright\left[R_{3}\right] M_{s g}^{1}(a, b, v @ c)\left|\left\langle R_{1}^{t_{k+1}}\right\rangle \llbracket P\right| N_{1} \rrbracket\left|\left\langle R_{2}^{\prime t_{k+1}}\right\rangle \llbracket N_{2} \rrbracket\right| \\
& \left\langle R_{3}^{\prime t_{k+1}}\right\rangle \llbracket N_{3} \rrbracket\left|\left\langle R_{4}{ }^{t_{k+1}}\right\rangle \llbracket \llbracket N_{4} \rrbracket\right|\left\langle R_{5}^{\prime t_{k+1}}\right\rangle \llbracket Q \mid N_{5} \rrbracket
\end{aligned}
$$

Further suppose $\left\langle R_{3}^{\prime t_{k+1}}\right\rangle(b)=R_{4}$ and the message $\left[R_{3}\right] M_{s g}^{1}(a, b, v @ c)$ is propagated to $R_{4}$. Since $\left(R_{3}, R_{4}\right) \in \Gamma_{c}$. Therefore again using the rule (R-MSG-FWD) the configuration

$$
\begin{gathered}
\Gamma_{c} \triangleright\left[R_{3}\right] M_{s g}^{1}(a, b, v @ c)\left|\left\langle R_{1}^{t_{k+1}}\right\rangle \llbracket P\right| N_{1} \rrbracket\left|\left\langle R_{2}^{\prime t_{k+1}}\right\rangle \llbracket N_{2} \rrbracket\right| \\
\left\langle R_{3}^{t^{\prime}}{ }^{\prime+1}\right\rangle \llbracket N_{3} \rrbracket\left|\left\langle R_{4}{ }^{t_{k+1}}\right\rangle \llbracket N_{4} \rrbracket\right|\left\langle R_{5}^{t_{k+1}}\right\rangle \llbracket Q \mid N_{5} \rrbracket
\end{gathered}
$$

reduces to

$$
\begin{gathered}
\Gamma_{c} \triangleright\left[R_{4}\right] M_{s g}^{2}(a, b, v @ c)\left|\left\langle R_{1} t_{k+1}\right\rangle \llbracket\right| P\left|N_{1} \rrbracket\right|\left\langle R_{2}^{\prime t_{k+1}}\right\rangle \llbracket \llbracket N_{2} \rrbracket \mid \\
\left\langle R_{3}^{\prime t_{k+1}}\right\rangle \llbracket N_{3} \rrbracket\left|\left\langle R_{4}{ }^{t_{k+1}}\right\rangle \llbracket N_{4} \rrbracket\right|\left\langle R_{5}^{\prime t_{k+1}}\right\rangle \llbracket Q \mid N_{5} \rrbracket
\end{gathered}
$$

Similarly again all the tables are updated with new entries with an application of rule (R-TABLE-UPDATE) at global clock $t_{k+2}$. Further suppose $\left\langle R_{4}^{\prime t_{k+2}}\right\rangle(b)=R_{5}$ and message $\left[R_{4}\right] M_{s g}^{2}(a, b, v @ c)$ is propagated to $R_{5}$. Since $\left(R_{4}, R_{5}\right) \in \Gamma_{c}$. Therefore again using the rule (R-MSG-FWD) the configuration

$$
\begin{aligned}
& \Gamma_{c} \triangleright\left[R_{4}\right] M_{s g}^{2}(a, b, v @ c)\left|\left\langle R_{1}^{\prime t_{k+2}}\right\rangle \llbracket P\right| N_{1} \rrbracket\left|\left\langle R_{2}^{\prime \prime t_{k+2}}\right\rangle \llbracket N_{2} \rrbracket\right| \\
& \left\langle R_{3}^{\prime \prime t_{k+2}}\right\rangle \llbracket N_{3} \rrbracket\left|\left\langle R_{4}^{\prime t_{k+2}}\right\rangle \llbracket N_{4} \rrbracket\right|\left\langle R_{5}^{\prime t_{k+2}}\right\rangle \llbracket Q \mid N_{5} \rrbracket
\end{aligned}
$$

reduces to

$$
\begin{gathered}
\Gamma_{c} \triangleright\left[R_{5} \rrbracket M_{s g}^{3}(a, b, v @ c)\left|\left\langle R_{1}^{\prime t_{k+2}}\right\rangle \llbracket\right| P\left|N_{1} \rrbracket\right|\left\langle R_{2}^{\prime \prime t_{k+2}}\right\rangle \llbracket \llbracket N_{2} \rrbracket \mid\right. \\
\left\langle R_{3}^{\prime \prime t_{k+2}}\right\rangle \llbracket N_{3} \rrbracket\left|\left\langle R_{4}^{\prime \prime}{ }^{\prime} k_{k+2}\right\rangle \llbracket N_{4} \rrbracket\right|\left\langle R_{5}^{\prime \prime t_{k+2}}\right\rangle \llbracket Q \mid N_{5} \rrbracket
\end{gathered}
$$

Because $\left\langle R_{5}^{\prime t_{k+2}}\right\rangle(b)=R_{5}$, the value $v$ is delivered to the waiting process at $b$ using the rule (R-COMM). Therefore the configuration

$$
\begin{gathered}
\Gamma_{c} \triangleright\left[R_{5}\right] M_{s g}^{3}(a, b, v @ c)\left|\left\langle R_{1}^{\prime t_{k+2}}\right\rangle \llbracket P\right| N_{1} \rrbracket\left|\left\langle R_{2}^{\prime \prime t_{k+2}}\right\rangle \llbracket N_{2} \rrbracket\right| \\
\quad\left\langle R_{3}^{\prime \prime t_{k+2}}\right\rangle \llbracket N_{3} \rrbracket\left|\left\langle R_{4}^{\prime t_{k+2}}\right\rangle \llbracket N_{4} \rrbracket\right|\left\langle R_{5}^{\prime t_{k+2}}\right\rangle \llbracket b[c ?(x) R] \mid N_{5} \rrbracket
\end{gathered}
$$

reduces to

$$
\begin{aligned}
& \Gamma_{c} \triangleright\left\langle R_{1}^{\prime t_{k+2}}\right\rangle \llbracket P\left|N_{1} \rrbracket\right|\left\langle R_{2}^{\prime \prime t_{k+2}}\right\rangle \llbracket N_{2} \rrbracket\left|\left\langle R_{3}^{\prime \prime \prime} t_{k+2}\right\rangle \llbracket N_{3} \rrbracket\right| \\
& \left\langle R_{4}^{\prime t_{k+2}}\right\rangle \llbracket N_{4} \rrbracket\left|\left\langle R_{5}^{\prime t_{k+2}^{2}}\right\rangle \llbracket b[R\{v / x\}]\right| N_{5} \rrbracket
\end{aligned}
$$

Similarly all the tables are updated with new entries by rule (R-TABLE-UPDATE) at every global clock $t_{k^{\prime}}$ where $t_{k^{\prime}}=t_{k}, t_{k+1}, t_{k+2} \ldots \ldots$. Thus all the routers in a path of communication between $R_{1}$ and $R_{5}$ are updated dynamically. This method of routing table update is known as distance vector routing updates.

Previously the path for communication from $a$ to $b$ is via $R_{1} \rightsquigarrow R_{3} \rightsquigarrow R_{5}$ where the value propagating message hops two routers before delivering the value at the destination process which means paths are fixed. But now path are changed and new path for communication from $a$ to $b$ via $R_{1} \rightsquigarrow R_{3} \rightsquigarrow R_{4} \rightsquigarrow R_{5}$. Due to this all the routing tables are updated dynamically. Therefore paths are also changed and this ensures the best optimal path. This is more closer to the real distributed network. 


\section{WELl FORMED CONFIGURATIONS}

We define a set of conditions on well formed configurations and prove them in $\mathrm{DR}_{\pi}^{\varphi}$. The well formedness is preserved under reductions. The conditions on well formed configurations are explained in definition 1 and $\mathrm{DR}_{\pi}^{\varphi}$ is ensured by the reduction semantics.

In definition 1, the concept of well formed configurations in $\mathrm{DR}_{\pi}^{\varphi}$ is inherited from [8] and the reduction rule (6) and (7) are used to illustrate that when (R-COMM) and (R-MSG-FWD) occurs, reduction rule (R-TABLE-UPDATE) is prohibited for given network and vice- versa. These configuration rules will prevent looping and congestion in the network. Hence it will reduce inconsistency in the network.

Definition 1: well formed configuration A configuration is called well formed if it satisfies the following conditions:

1) $\Gamma_{c} \triangleright \varepsilon$ is a well formed system.

2) If $\Gamma_{c} \triangleright\left\langle R^{t_{k^{\prime}}}\right\rangle \llbracket N \rrbracket \mid S$ is well formed at a global clock if

a) $\quad \Gamma_{c} \triangleright S$ is well formed where $S$ contains no message at $R$.

b) $\left\langle R^{t} k^{\prime}\right\rangle$ does not occur in $S$. (Uniqueness of router name $R$ )

c) $\forall \in \operatorname{fn}(N)$ such that $m \in N N$ where $N N$ is the set of node names, if $\left\langle R^{t_{k^{\prime}}}\right\rangle(m)=R$ then $\forall\left\langle R_{1}^{t_{k^{\prime}}}\right\rangle \in S,\left\langle R_{1}^{t_{k^{\prime}}}\right\rangle(m) \neq R_{1}$. (Uniqueness of node name $m)$

3) If $\Gamma_{c} \triangleright\left\langle R^{t_{k^{\prime}}}\right\rangle \llbracket N \rrbracket \mid S$ is well formed at a global clock $t=t_{k^{\prime}}, t_{k^{\prime}+1},$. then $\Gamma_{c} \triangleright\left\langle R^{t_{k^{\prime}+1}}\right\rangle \llbracket N \rrbracket \mid S$ is also well formed.

4) $\quad \Gamma_{c} \triangleright[R] M_{s g}^{h}(n, m, v @ c) \mid S$ is a well formed if

a) $\quad \Gamma_{c} \triangleright S$ is well formed and $S \equiv\left\langle R^{t_{k^{\prime}}}\right\rangle \llbracket N \rrbracket \mid S^{\prime}$ for some $S^{\prime}$

b) There exists a path $P\left(R^{\prime}, R\right)=R^{\prime} \rightsquigarrow R^{\prime \prime} \rightsquigarrow$ ....R for some $R^{\prime}, R^{\prime \prime}, \ldots \ldots$

such that $\left\langle R^{\prime t} k_{k^{\prime}}\right\rangle(n)=R^{\prime}$ and $\left\langle R^{\prime t_{k^{\prime}}}\right\rangle(m)=$ $R^{\prime \prime} \ldots$. where $h=\left|\rho\left(R^{\prime}, R\right)\right|-1$

5) In any well formed configuration $\Gamma_{c} \triangleright S$, for every pair of nodes $\mathrm{n}$ and $\mathrm{m}$ such that $\left\langle R_{i}{ }^{{ }^{\prime}{ }^{\prime}}\right\rangle(n)=$ $R_{i}$ and $\left\langle R_{j}{ }^{{ }^{\prime}}{ }^{\prime}\right\rangle(m)=R_{j}$ at any global clock $t=t_{k^{\prime}}$ where $\left(R_{i}, R_{j}\right) \in S$, there exists a unique path $R_{i} \rightsquigarrow R_{j}$ such that

$\left\langle R_{i}{ }^{t} k_{k^{\prime}}\right\rangle(m)=R^{\prime},\left\langle R^{\prime t_{k^{\prime}+1}}\right\rangle(m)=R^{\prime \prime}, \ldots \ldots . . R^{\prime t_{k^{\prime}+p}}$ \rangle$(m)=R_{j}$

6) $\quad \Gamma_{c} \triangleright S$ is well formed iff

a) If $\Gamma_{c} \triangleright S \longrightarrow^{h} \Gamma_{c} \triangleright S^{\prime}$ is using rule (RCOMM) then $\Gamma_{c} \triangleright S \nrightarrow{ }^{h} \Gamma_{c} \triangleright S^{\prime}$ will not be used rule (R-TABLE-UPDATE).

b) If $\Gamma_{c} \triangleright S \longrightarrow{ }^{h} \Gamma_{c} \triangleright S^{\prime}$ is using rule (R-MSGFWD) then $\Gamma_{c} \triangleright S \nrightarrow{ }^{h} \Gamma_{c} \triangleright S^{\prime}$ will not be used rule (R-TABLE-UPDATE).

7) $\quad \Gamma_{c} \triangleright S$ is well formed iff

a) If $\Gamma_{c} \triangleright S \longrightarrow^{h} \Gamma_{c} \triangleright S^{\prime}$ is using rule (RTABLE-UPDATE) then

$\Gamma_{c} \triangleright S \nrightarrow{ }^{h} \Gamma_{c} \triangleright S^{\prime}$ will not be used either rule (R-COMM) or rule (R-MSG-FWD) .

b) If $\Gamma_{c} \triangleright S \longrightarrow{ }^{h} \Gamma_{c} \triangleright S^{\prime}$ is using rule (RTABLE-UPDATE) then

$\Gamma_{c} \triangleright S \nrightarrow{ }^{h} \Gamma_{c} \triangleright S^{\prime}$ will not be used rule (RCOMM) and rule (R-MSG-FWD) .
Lemma.1. Suppose $S \equiv T$ then $\Gamma_{c} \triangleright S$ is well formed iff $\Gamma_{c} \triangleright T$ is well formed.

Proof.(OUTLINE) By induction on definition of $\equiv$.

Theorem 1. If $\Gamma_{c} \triangleright S$ is well formed configuration and $\Gamma_{c} \triangleright S \longrightarrow^{h} \Gamma_{c} \triangleright S^{\prime}$ then $\Gamma_{c} \triangleright S^{\prime}$ is also well formed.

Proof. (OUTLINE) By rule induction on inference of $\Gamma_{c} \triangleright S \longrightarrow{ }^{h} \Gamma_{c} \triangleright S^{\prime}$ then $\Gamma_{c} \triangleright S^{\prime}$. It is easy to prove that each inference of $\Gamma_{c} \triangleright S^{\prime}$, using the reduction rules in Fig. 7, satisfies all the properties of a well formed configuration.

\section{EQUIVALENCE BETWEEN DR ${ }_{\pi}^{\varphi}$ AND $\mathrm{D}_{\pi}$}

We proved that whenever a $D_{\pi}$ [2] system does a reduction there exists a corresponding well formed configuration in $\mathrm{DR}_{\pi}^{\varphi}$ which can do a number of reductions such that the residual are equivalent upto structural equivalence after $\phi$ abstraction of the residual system in $\mathrm{DR}_{\pi}^{\varphi}$. Similarly for the converse, we proved that whenever a well formed configuration in $\mathrm{DR}_{\pi}^{\varphi}$ does a reduction there exists a corresponding $\mathrm{D}_{\pi}$ system which either does nothing or does a reduction where residuals of both $\mathrm{D}_{\pi}$ and $\mathrm{DR}_{\pi}^{\varphi}$ systems are matched upto structural equivalence. Since $D_{\pi}$ is a specification for $\mathrm{DR}_{\pi}^{\varphi}$ therefore we have shown that $\mathrm{DR}_{\pi}^{\varphi}$ conforms to its specification. Our model is also closer to real distributed networks.

we define a function to abstract away the details of routers and paths from a $\mathrm{DR}_{\pi}^{\varphi}$ term state theorems about the equivalence of $\mathrm{DR}_{\pi}^{\varphi}$ with $\mathrm{D}_{\pi}$.

Definition 2: We define a function $\phi: L S Y \rightarrow H S Y$, where $L S Y$ and $H S Y$ are sets of $\mathrm{DR}_{\pi}^{\varphi}$ system terms and $\mathrm{D}_{\pi}$ systems respectively, as follows:

$$
\begin{array}{ll}
\phi(\varepsilon) & =n i l \\
\phi\left(\left\langle R^{t_{k^{\prime}}}\right\rangle \llbracket N \rrbracket\right) & = \\
\phi\left([R] M_{s g}^{h}(n, m, v @ c)\right) & =n[m !\langle v @ c\rangle] \\
\phi(S \mid T) & =\phi(S) \mid \phi(T)
\end{array}
$$

Proposition 1. For any system term $L$ in $\mathrm{DR}_{\pi}^{\varphi}$ such that $\phi(L)=H$ and $H \equiv H^{\prime}$ implies that there exists some system term $L^{\prime}$ in $\mathrm{DR}_{\pi}^{\varphi}$ such that $\phi\left(L^{\prime}\right)=H^{\prime}$ and $L \equiv L^{\prime}$.

Proof. We shall prove it by induction on various forms $L$ can take and syntactic analysis of $L$ such that $\phi(L)=H$ and $H \equiv H^{\prime}$

1) Let us take a case when a system $L$, in $\mathrm{DR}_{\pi}^{\varphi}$, is of the form

$L \equiv\left\langle R_{1}{ }^{{ }^{k^{\prime}}}\right\rangle \llbracket n[m !\langle v @ c\rangle \mid P] \mid N \rrbracket$

By using $\phi$ definition, we get

$$
\begin{gathered}
\phi(L)=n[m !\langle v @ c\rangle \mid P] \mid N \\
H \equiv n[m !\langle v @ c\rangle \mid P] \mid N
\end{gathered}
$$

Therefore when we write $\phi(L)=H$ for some term $L$ in $\mathrm{DR}_{\pi}^{\varphi}$ and a $\mathrm{D}_{\pi}$ system $H$, we can rearrange the terms in a $\mathrm{D}_{\pi}$ systems $H^{\prime}$, by using various axioms of structural equivalence (SE-COM) and (SE-ID). Since

$$
\begin{gathered}
H^{\prime} \equiv N \mid n[m !\langle v @ c\rangle \mid P] \\
\text { or } \\
H^{\prime} \equiv n[m !\langle v @ c\rangle \mid P]|N| e
\end{gathered}
$$

Therefore $H \equiv H^{\prime}$. When a system term $L^{\prime}$, in $\mathrm{DR}_{\pi}^{\varphi}$, is of the form

$$
L^{\prime} \equiv\left\langle R_{1}{ }_{k^{\prime}}\right\rangle \llbracket N \mid n[m !\langle v @ c\rangle \mid P] \rrbracket
$$


Further by definition of $\phi$ we get,

$$
\phi\left(L^{\prime}\right)=N\left|n[m !\langle v @ c\rangle \mid P] H^{\prime} \equiv N\right| n[m !\langle v @ c\rangle \mid P]
$$

By using axiom (SE-COM), we get

$$
H^{\prime} \equiv n[m !\langle v @ c\rangle \mid P] \mid N \equiv H
$$

Now it is clear that, the relation $=$ in the definition $\phi$ is much stronger than $\equiv$ i.e. $\phi$ is closed upto $\equiv$. Therefore, by definition of $\equiv$ given in Figure 3 it can be easily verified that $\phi\left(L^{\prime}\right)=H^{\prime}$ and $L \equiv L^{\prime}$.

2) Let us take another case when a system $L$, in $\mathrm{DR}_{\pi}^{\varphi}$, is of the form

$$
L \equiv\left[R_{1}\right] M_{s g}^{0}(n, m, v @ c) \mid\left\langle R_{2}{ }^{t_{k^{\prime}}} \llbracket N \rrbracket\right| M
$$

By using $\phi$ definition on $L$, we get

$$
\begin{gathered}
\phi(L)=n[m !\langle v @ c\rangle \mid P]|N| M \\
H \equiv n[m !\langle v @ c\rangle \mid P]|N| M
\end{gathered}
$$

Therefore when we write $\phi(L)=H$, for some term $L$ in $\mathrm{DR}_{\pi}^{\varphi}$ and $\mathrm{D}_{\pi}$ system $H$, we can rearrange the terms in a $\mathrm{D}_{\pi}$ systems $H^{\prime}$, by using various axioms of structural equivalence (SE-COM),(SE-ASSOC) and (SE-ID). Since $H^{\prime}$ is take various forms like,

$$
\begin{gathered}
H^{\prime} \equiv n[m !\langle v @ c\rangle \mid P]|M| N \\
\text { or } \\
H^{\prime} \equiv M|N| n[m !\langle v @ c\rangle \mid P] \\
\text { or } \\
H^{\prime} \equiv n[m !\langle v @ c\rangle \mid P]|N| M \mid e
\end{gathered}
$$

All the form of $H^{\prime}$ is structurally equivalent to $H$, by using various axioms of structural equivalence. Since $H^{\prime}$

Now we take system term $L^{\prime}$ in $\mathrm{DR}_{\pi}^{\varphi}$, is the form of

$$
L^{\prime} \equiv\left\langle R_{2}{ }_{k^{\prime}} \llbracket N \rrbracket|M|\left[R_{1}\right] M_{s g}^{0}(n, m, v @ c)\right.
$$

Further by definition of $\phi$ we get,

$$
\begin{gathered}
\phi\left(L^{\prime}\right)=N|| M \mid n[m !\langle v @ c\rangle \mid P] \\
H^{\prime} \equiv N|| M \mid n[m !\langle v @ c\rangle \mid P]
\end{gathered}
$$

By using rule (SE-COM), we get

$$
H^{\prime} \equiv n[m !\langle v @ c\rangle \mid P]|N| M \equiv H
$$

Therefore $\phi\left(L^{\prime}\right)=H^{\prime}$ and $L \equiv L^{\prime}$.

Similarly other cases can be proved.

Proposition 2. For any system term $L$ in $\mathrm{DR}_{\pi}^{\varphi} L \equiv L^{\prime}$ implies $\phi(L) \equiv \phi\left(L^{\prime}\right)$.

Proof. This can be proved by induction on the definition of $L$ and $\equiv$ as defined in Figure 2 and . By applying function $\phi$ above proposition can be derived fairly straightforward.

Lemma.2. In a $\mathrm{D}_{\pi}$ system $H_{1}$ does a reduction $H_{1} \longrightarrow$ $H_{2}$ and $\phi\left(L_{1}\right)=H_{1}^{\prime}$ such that $H_{1}^{\prime} \equiv H_{1}$ where $L_{1}$ is a system term over a well formed configuration $\Gamma_{c} \triangleright L_{1}$ in $\mathrm{DR}_{\pi}^{\varphi}$, then $\Gamma_{c} \triangleright L_{1} \longrightarrow \Gamma_{c} \triangleright L_{2}$ for some $h$ such that $\phi\left(L_{2}\right)=H_{2}^{\prime}$ where $H_{2}^{\prime} \equiv H_{2}$.

Proof. We shall prove it by rule induction on the inference of a $\mathrm{D}_{\pi}$ system reduction $H_{1} \longrightarrow H_{2}$ and syntactic analysis of $L_{1}$ such that $\phi\left(L_{1}\right)=H_{1}^{\prime}$ where $H_{1}^{\prime} \equiv H_{1}$. There are various possibilities and we we will take each of them as follows:

1) Let us take a case where a $\mathrm{D}_{\pi}$ system $H_{1}$ is the form $l_{1}[c ?(x) P$

$\mid M] \mid l_{2}\left[l_{1} !\langle v @ c\rangle \mid N\right]$. Suppose the $\mathrm{D}_{\pi}$ system $H_{1}$ does a reduction to

$$
l_{1}[P\{v / x\} \mid M] \mid l_{2}[N]
$$

By using the rule (R-H-COMM) where

$$
H_{2} \equiv l_{1}[P\{v / x\} \mid M] \mid l_{2}[N]
$$

A system term $L_{1}$ in $\mathrm{DR}_{\pi}^{\varphi}$, such that $\phi\left(L_{1}\right)=H_{1}^{\prime}$ can take various forms. We shall examine each of them as follows:

a) We take the case where $L_{1}$ is structurally equivalent to

$\left\langle R_{1}^{t_{k^{\prime}}}\right\rangle \llbracket l_{1}[c ?(x) P \mid M] \rrbracket \mid\left\langle R_{2}^{t_{k^{\prime}}}\right\rangle \llbracket l_{2}\left[l_{1} !\langle v @ c\rangle \mid N\right] \rrbracket$ for some $R_{1}$ and $R_{2}$. We can clearly see that $\phi\left(L_{1}\right)=l_{1}[c ?(x) P \mid M] \mid l_{2}\left[l_{1} !\langle v @ c\rangle \mid N\right]$ where

$$
\begin{aligned}
l_{1}[c ?(x) P \mid M] \mid & l_{2}\left[l_{1} !\langle v @ c\rangle \mid N\right] \equiv H_{1} \text { s.t. } \\
& H_{1}^{\prime} \equiv H_{1}
\end{aligned}
$$

We know that $\Gamma_{c} \triangleright L_{1}$ is a well formed system and therefore $L_{1}$ does a following reduction using rule (R-OUT) to become

$$
\begin{gathered}
{[R] M_{s g}^{0}\left(l_{2}, l_{1}, v @ c\right)\left|\left\langle R_{2}^{t_{k^{\prime}}}\right\rangle \llbracket l_{2}[N] \rrbracket\right|\left\langle R_{1}^{t_{k^{\prime}}}\right\rangle} \\
\llbracket l_{1}[c ?(x) P \mid M] \rrbracket
\end{gathered}
$$

We use various standard axioms of structural equivalence rules and by definition of $\phi$ we know that

$\phi\left(L_{2}\right)=l_{2}\left[l_{1} !\langle v @ c\rangle\right]\left|l_{2}[N]\right| l_{1}[c ?(x) P \mid M]$ where

$$
H_{2}^{\prime} \equiv l_{2}\left[l_{1} !\langle v @ c\rangle\right]\left|l_{2}[N]\right| l_{1}[c ?(x) P \mid M]
$$

By using axiom (R-H-COM),we get

$$
H_{2}^{\prime} \equiv l_{2}\left[l_{1} !\langle v @ c\rangle\right]\left|l_{1}[c ?(x) P \mid M]\right| l_{2}[N]
$$

By using axiom (R-H-COMM), we get

$$
H_{2}^{\prime} \equiv l_{1}[P\{v / x\} \mid M] \mid l_{2}[N] \equiv H_{2}
$$

Further as we know that $\Gamma_{c} \triangleright L_{1}$ is a well formed system and therefore according to the condition of well formed configuration $R_{2} \rightsquigarrow$ $R_{1}$ where $\left\langle R_{2}^{t_{k^{\prime}}}\right\rangle\left(l_{2}\right)=R_{2}$ and $\left\langle R_{1}^{t_{k^{\prime}}}\right\rangle\left(l_{1}\right)=R_{1}$. Let us assume that $\left\langle R_{2}^{t_{k^{\prime}}}\right\rangle\left(l_{2}\right)=R_{3}$ for some $R_{3}$ such that $\left(R_{2}, R_{3}\right) \in \Gamma_{c}$. A reduction is done using rule (R-MSG-FWD)

$$
\begin{gathered}
{\left[R_{2}\right] M_{s g}^{0}\left(l_{2}, l_{1}, v @ c\right)\left|\left\langle R_{2}^{t_{k^{\prime}}}\right\rangle \llbracket l_{2}[N] \rrbracket\right|\left\langle R_{1}^{t_{k^{\prime}}}\right\rangle} \\
\qquad l_{1}\left[\left.c ?(x) P\right|^{M}\right] \rrbracket \\
\text { does a reduction to } \\
\quad\left[R_{3}\right] M_{s g}^{1}\left(l_{2}, l_{1}, v @ c\right)\left|\left\langle R_{2}^{t_{k^{\prime}}}\right\rangle \llbracket l_{2}[N] \rrbracket\right|\left\langle R_{1}^{t_{k^{\prime}}}\right\rangle \\
\qquad l_{1}\left[\left.c ?(x) P\right|^{M}\right] \rrbracket
\end{gathered}
$$

where

$$
\begin{aligned}
& \phi\left(L_{2}\right)=l_{2}\left[l_{1} !\langle v @ c\rangle\right]\left|l_{2}[N]\right| l_{1}[c ?(x) P \mid M] \\
& \text { and } \\
& \qquad H_{2}^{\prime} \equiv l_{2}\left[l_{1} !\langle v @ c\rangle\right]\left|l_{2}[N]\right| l_{1}[c ?(x) P \mid M]
\end{aligned}
$$

By using axiom (S-MONOID-COM), we get

$$
H_{2}^{\prime} \equiv l_{2}\left[l_{1} !\langle v @ c\rangle\right]\left|l_{1}[c ?(x) P \mid M]\right| l_{2}[N]
$$

By using axiom (R-H-COMM), we get

$$
H_{2}^{\prime} \equiv l_{1}[P\{v / x\} \mid M] \mid l_{2}[N] \equiv H_{2}
$$

By using rule (R-COMM), after reduction directly gives the form of $\mathrm{H}_{2}$.

b) We can take another possibility of the form that a system $L_{1}$ in $\mathrm{DR}_{\pi}^{\varphi}$ can take. In a $\mathrm{D}_{\pi}$ system $H_{1}$ is the form $l_{1}[c ?(x) P \mid M] \mid l_{2}\left[l_{1} !\langle v @ c\rangle \mid N\right]$. It is possible that $M$ and $N$ contain several output 
process terms. Theses output terms will have equivalent messages terms at $L_{1}$ which are originated at nodes $l_{1}$ and $l_{2}$ to carry arbitrary values to some channel at various nodes. Therefore $L_{1}$ may contain several messages which may be equivalent to one of the output terms in $M$ or $N$ after $\phi$ abstraction.

2) We will now consider another possibility when a $D_{\pi}$ system $H_{1}$ is the form

$$
n[\text { if } v=v \text { then } P \text { else } Q]
$$

and does a reduction using (R-H-MATCH), we get

$$
n[\text { if } v=v \text { then } P \text { else } Q] \rightarrow n[P]
$$

where $n[P] \equiv \mathrm{H}_{2}$

In one possibility a system term $L_{1}$, in $\mathrm{DR}_{\pi}^{\varphi}$, can take a form

$$
\left\langle R^{t_{k^{\prime}}}\right\rangle \llbracket n[\text { if } v=v \text { then } P \text { else } Q] \rrbracket \equiv L_{1}
$$

for some $R$ such that

$$
\phi\left(L_{1}\right)=n[\text { if } v=v \text { then } P \text { else } Q]
$$

with an application of rule (R-MATCH) in a well formed configuration $\Gamma_{c} \triangleright L_{1}$ can do a reduction to

$$
\Gamma_{c} \triangleright\left\langle R^{t_{k^{\prime}}}\right\rangle \llbracket n[P] \rrbracket
$$

Here $L_{2} \equiv\left\langle R^{t_{k^{\prime}}}\right\rangle \llbracket n[P] \rrbracket$. Further with an application of the function $\phi$ on $L_{2}$ we can get

$$
\phi\left(L_{1}\right)=n[P] \equiv H_{2}^{\prime}
$$

3) We will now consider another possibility when a $D_{\pi}$ system $H_{1}$ is the form

$$
n\left[\text { if } v_{1}=v_{2} \text { then } P \text { else } Q\right]
$$

and does a reduction using (R-H-MISMATCH), we get

$$
n[\text { if } v=v \text { then } P \text { else } Q] \rightarrow n[Q]
$$

where $n[Q] \equiv H_{2}$

In one possibility a system term $L_{1}$, in $\mathrm{DR}_{\pi}^{\varphi}$, can take a form

$$
\left\langle R^{t_{k^{\prime}}}\right\rangle \llbracket n\left[\text { if } v_{1}=v_{2} \text { then } P \text { else } Q\right] \rrbracket \equiv L_{1}
$$

for some $R$ such that

$$
\phi\left(L_{1}\right)=n\left[\text { if } v_{1}=v_{2} \text { then } P \text { else } Q\right]
$$

with an application of rule (R-MISMATCH) in a well formed configuration $\Gamma_{c} \triangleright L_{1}$ can do a reduction to

$$
\Gamma_{c} \triangleright\left\langle R^{t_{k^{\prime}}}\right\rangle \llbracket n[Q] \rrbracket
$$

Here $L_{2} \equiv\left\langle R^{t_{k^{\prime}}}\right\rangle \llbracket n[Q] \rrbracket$. Further with an application of the function $\phi$ on $L_{2}$ we can get

$$
\phi\left(L_{1}\right)=n[Q] \equiv H_{2}^{\prime}
$$

4) Now we consider the cases of compositional reduction of a $\mathrm{D}_{\pi}$ system. Let us assume that a $\mathrm{D}_{\pi}$ system $H_{1}$ is of the form $P_{1} \mid P_{2}$. An application of the rule (R-H-CONTX) reduces $H_{1}$ to $P_{1}^{\prime} \mid P_{2}$. Let us assume that a system term in $\mathrm{DR}_{\pi}^{\varphi}$, is of the form $L_{1} \mid L_{2}$ such that $\phi\left(L_{1}\right)=P_{1}$ and $\phi\left(L_{2}\right)=P_{2}$. We also assume that a configurations $\Gamma_{c} \triangleright L_{1} \mid L_{2}$ and $\Gamma_{c} \triangleright L_{1}$ are well formed configurations. By induction we can say that $\Gamma_{c} \triangleright L_{1} \longrightarrow \Gamma_{c} \triangleright L_{1}^{\prime}$ such that $\phi\left(L_{1}^{\prime}\right)=P_{1}^{\prime \prime}$ for some $h, P_{1}^{\prime \prime} \equiv P_{1}^{\prime}$. Therefore using the rule (R-CONTX), we can conclude that $\Gamma_{c} \triangleright L_{1}\left|L_{2} \longrightarrow \Gamma_{c} \triangleright L_{1}^{\prime}\right| L_{2}$. We know that $\phi\left(L_{1}^{\prime} \mid L_{2}\right)=\phi\left(L_{1}^{\prime}\right) \mid \phi\left(L_{2}\right)$. Since $\phi\left(L_{2}\right)=P_{2}$ and $\phi\left(L_{1}^{\prime}\right)=P_{1}^{\prime \prime}$ therefore $\phi\left(L_{1}^{\prime} \mid L_{2}\right)=$ $P_{1}^{\prime \prime} \mid P_{2}$. Further we already know that $P_{1}^{\prime \prime} \equiv P_{1}^{\prime}$ therefore from the axioms of structural equivalence , we can conclude that $P_{1}^{\prime} \mid P_{2} \equiv H_{2}$.

Same as $H_{1}$ is the form of $P_{2} \mid P_{1}$. We can proved similarly.

5) Let us now consider the last case when a $D_{\pi}$ system $H_{1}$ does a reduction to $H_{2}$ using the rule (R-HSTRUCT) because $H_{1}^{\prime} \longrightarrow H_{2}^{\prime}$ where $H_{1} \equiv H_{1}^{\prime}$ and $H_{2} \equiv H_{2}^{\prime}$. Let us assume that for a system $L_{1}$ in $\operatorname{DR}_{\pi}^{\varphi}$, $\phi\left(L_{1}\right)=H_{1}$. We also assume that $\Gamma_{c} \triangleright L_{1}$ is a well formed configuration. Since $H_{1} \equiv H_{1}^{\prime}$ therefore using proposition 1 . we can say that there exists a $L_{1}^{\prime}$ such that $\phi\left(L_{1}^{\prime}\right)=H_{1}^{\prime}$ and $L \equiv L^{\prime}$. Further using lemma.1. We know that $\Gamma_{c} \triangleright L_{1}^{\prime}$ is a well formed. Now by induction we can say $H_{1}^{\prime} \longrightarrow H_{2}^{\prime}$ implies $\Gamma_{c} \triangleright L_{1}^{\prime} \longrightarrow$ $\Gamma_{c} \triangleright L_{1}^{\prime \prime}$ for some $L_{1}^{\prime \prime}$ and $h$ such that $\phi\left(L_{1}^{\prime \prime}\right)=H_{2}^{\prime \prime}$ for some $H_{2}^{\prime \prime}$ such that $H_{2}^{\prime \prime} \equiv H_{2}^{\prime}$. We already know that $L_{1} \equiv L_{1}^{\prime}$ therefore with an application of rule (RSTRUCT). We can say that $\Gamma_{c} \triangleright L_{1} \stackrel{*}{\longrightarrow} \Gamma_{c} \triangleright L_{1}^{\prime \prime}$. We know that $\phi\left(L_{1}^{\prime \prime}\right)=H_{2}^{\prime \prime}$ and since $H_{2}^{\prime \prime} \equiv H_{2}^{\prime}, H_{2}^{\prime} \equiv H_{2}$ therefore $H_{2}^{\prime \prime} \equiv H_{2}$

Lemma.3.In $\mathrm{DR}_{\pi}^{\varphi}$, if a well formed configuration $\Gamma_{c} \triangleright L_{1}$ does a reduction $\Gamma_{c} \triangleright L_{1} \longrightarrow \Gamma_{c} \triangleright L_{2}$ and $\phi\left(L_{1}\right)=H_{1}$ where $H_{1}$ is a $\mathrm{D}_{\pi}$ system then either there exists a $\mathrm{D}_{\pi}$ system $H_{2}$ such that $H_{1} \longrightarrow H_{2}$ and $\phi\left(L_{2}\right) \equiv H_{2}$ or $\phi\left(L_{2}\right) \equiv H_{1}$.

Proof. By induction on the inference of reduction $\Gamma_{c} \triangleright$ $L_{1} \longrightarrow \Gamma_{c} \triangleright L_{1}$ of well formed configurations in $\mathrm{DR}_{\pi}^{\varphi}$ and syntactic analysis of $\phi\left(L_{1}\right)=H_{1}$. There are various possibilities and we we will take each of them as follows:

1) Let us take a case when a system $L_{1}$, in $\operatorname{DR}_{\pi}^{\varphi}$, is of the form $\left\langle R^{t_{k^{\prime}}}\right\rangle \llbracket n[m !(v @ c) \mid P] \mid N \rrbracket$. A well formed configuration $\Gamma_{c} \triangleright L_{1}$ does a reduction to

$$
\Gamma_{c} \triangleright[R] M_{s g}^{0}(n, m, v @ c)\left\langle R^{t_{k^{\prime}}}\right\rangle \llbracket n[P] \mid N \rrbracket
$$

using rule (R-OUT) in fig. 7 , Let a $\mathrm{D}_{\pi}$ system $H_{1}$ is of the form $n[m !(v @ c) \mid P] \mid N$ and by definition of $\phi$ we know that

$$
\begin{gathered}
\phi\left(L_{1}\right)=\phi\left(\left\langle R^{t_{k^{\prime}}}\right\rangle \llbracket n[m !(v @ c) \mid P] \mid N \rrbracket\right)= \\
n[m !(v @ c) \mid P] \mid N
\end{gathered}
$$

Further by definition of $\phi$ we know that

$$
\begin{gathered}
\phi\left(L_{2}\right)=\phi\left([R] M_{s g}^{0}(n, m, v @ c)\left\langle R^{t_{k^{\prime}}}\right\rangle \llbracket n[P] \mid N \rrbracket\right)= \\
n[m !(v @ c) \mid P] \mid N
\end{gathered}
$$

now by an application of axiom (S-H-MERGE), We can conclude that

$$
n[m !(v @ c) \mid P] \mid N \equiv H_{1}
$$

2) In another case we consider that a system term $L_{1}$, in $\mathrm{DR}_{\pi}^{\varphi}$, is of the form $\left[R_{1}\right] M_{s g}^{h}(n, m, v @ c) \mid\left\langle R_{2}{ }^{{ }_{k^{\prime}}} \llbracket N \rrbracket\right| S$. We consider a case when using the rule (R-MSGFWD), the well formed configuration $\Gamma_{c} \triangleright L_{1}$ does a reduction to $\Gamma_{c} \triangleright\left[R_{2}\right] M_{s g}^{h+1}$

$(n, m, v @ c) \mid\left\langle R_{2}{ }_{k^{\prime}} \llbracket N \rrbracket\right| S$. Let a $\mathrm{D}_{\pi}$ system $H_{1}$ be of the form $n[m !\langle v @ c\rangle]|N| S$ where $\phi\left(L_{1}\right)=H_{1}$. Clearly

$$
\begin{gathered}
\phi\left(L_{1}\right)=\phi\left(\left[R_{1}\right] M_{s g}^{h}(n, m, v @ c) \mid\left\langle R_{2} t_{k^{\prime}} \llbracket N \rrbracket\right| S\right) \\
\phi\left(L_{1}\right)=n[m !\langle v @ c\rangle]|N| S \equiv H_{1}
\end{gathered}
$$

Further by definition of $\phi$ we know that

$$
\begin{gathered}
\phi\left(L_{2}\right)=\phi\left(\left[R_{2}\right] M_{s g}^{h+1}(n, m, v @ c) \mid\left\langle R_{2}{ }^{t}{ }_{k^{\prime}} \llbracket N \rrbracket\right| S\right) \\
\phi\left(L_{2}\right)=n[m !\langle v @ c\rangle]|N| S \equiv H_{1}
\end{gathered}
$$

3) Now let us take a case when a system $L_{1}$, in $\mathrm{DR}_{\pi}^{\varphi}$, is of the form 
$[R] M_{s g}^{h}(n, m, v @ c)\left|\left\langle R_{k^{\prime}}^{t^{\prime}}\right\rangle \llbracket m[c ?(x) P \mid Q]\right| N \rrbracket . \quad$ The well formed configuration $\Gamma_{c} \triangleright L_{1}$ does a reduction using the rule (R-COMM) to another well formed configuration $\Gamma_{c} \triangleright\left\langle R_{k^{\prime}}\right\rangle \llbracket m[P\{v / x\} \mid Q] \mid N \rrbracket$. Let us assume that $\left.L_{2}=R^{t_{k^{\prime}}}\right\rangle \llbracket m[P\{v / x\} \mid Q] \mid N \rrbracket$. Clearly

$$
\phi\left(L_{1}\right)=n[m !\langle v @ c\rangle]|m[P\{v / x\} \mid Q]| N
$$

By using the rule (R-H-COMM), The $\mathrm{D}_{\pi}$ system $n[m !\langle v @ c\rangle]|m[P\{v / x\} \mid Q]| N$ can reduce to $m[P\{v / x\} \mid Q]|n[\varepsilon]| N$ which is structurally equivalent to $m[P\{v / x\} \mid Q] \mid N \equiv H_{1}$. We know that $\phi\left(L_{2}\right)=$ $m[P\{v / x\} \mid Q] \mid N \equiv H_{1}$

4) Now we take another case where a system term $L_{1}$, in $\mathrm{DR}_{\pi}^{\varphi}$, is of the form either $\Gamma_{c} \triangleright\left\langle R_{1}{ }_{k^{\prime}+1}\right\rangle[M] \mid S$ or $\Gamma_{c} \triangleright\left\langle R_{2}{ }_{k^{\prime}+1}\right\rangle[N] \mid T$. We can take one form $\Gamma_{c} \triangleright$ $\left\langle R_{1}{ }^{t}{ }^{\prime}+1\right\rangle[M] \mid S$. The well formed configuration $\Gamma_{c} \triangleright L_{1}$ reduces using the rule (R-TABLE-UPDATE) to another well formed configuration $\Gamma_{c} \triangleright\left\langle R_{1}^{\prime t_{k^{\prime}+1}}\right\rangle[M] \mid S$ and by definition of $\phi$ we know that

$$
\begin{aligned}
& \phi\left(L_{1}\right)=M \mid S \equiv H_{1} \\
& \phi\left(L_{2}\right)=M \mid S \equiv H_{1}
\end{aligned}
$$

5) Now we take another case where a system term $L_{1}$, in $\mathrm{DR}_{\pi}^{\varphi}$, is of the form $\Gamma_{c} \triangleright\left\langle R^{t_{k^{\prime}}}\right\rangle \llbracket n$ [if $v=$ $v$ then $P$ else $Q] \rrbracket$. The well formed configuration $\Gamma_{c} \triangleright L_{1}$ reduces using the rule (R-MATCH) to another well formed configuration $\Gamma_{c} \triangleright\left\langle R^{t} k^{\prime}\right\rangle \llbracket n[P] \rrbracket$ and by definition of $\phi$ we know that

$$
\begin{gathered}
\phi\left(L_{1}\right)=n[\text { if } v=v \text { then } P \text { else } Q] \\
\phi\left(L_{1}\right)=n[P] \equiv H_{1} \quad \text { where value is true then result } \\
\quad \text { is } P \\
\phi\left(L_{2}\right)=n[P] \equiv H_{1}
\end{gathered}
$$

6) Now we take another case where a system term $L_{1}$, in $\mathrm{DR}_{\pi}^{\varphi}$, is of the form $\Gamma_{c} \triangleright\left\langle R^{t_{k^{\prime}}}\right\rangle \llbracket n$ [if $v_{1} \neq$ $v_{2}$ then $P$ else $\left.Q\right] \rrbracket$. The well formed configuration $\Gamma_{c} \triangleright L_{1}$ reduces using the rule (R-MISMATCH) to another well formed configuration $\Gamma_{c} \triangleright\left\langle R^{t} k^{\prime}\right\rangle \llbracket n[Q] \rrbracket$ and by definition of $\phi$ we know that

$$
\begin{gathered}
\phi\left(L_{1}\right)=n\left[\text { if } v_{1} \neq v_{2} \text { then } P \text { else } Q\right]=n[Q] \equiv H_{1} \\
\phi\left(L_{1}\right)=n[Q] \equiv H_{1}
\end{gathered}
$$

7) Now let us take compositional cases. First suppose a system in in $\mathrm{DR}_{\pi}^{\varphi}$, is of the form $L_{1} \mid L_{2}$ and a $\mathrm{D}_{\pi}$ system is of the form $H_{1} \mid H_{2}$ where $\phi\left(L_{1}\right)=H_{1}$ and $\phi\left(L_{2}\right)=H_{2}$. By definition of the $\phi$ we can clearly see that $\phi\left(L_{1} \mid L_{2}\right)=H_{1} \mid H_{2}$. Now take the case when the well formed configuration in $\mathrm{DR}_{\pi}^{\varphi}, \Gamma_{c} \triangleright L_{1} \mid L_{2}$ does a reduction using the rule (R-CONTX) to another well formed configuration $\Gamma_{c} \triangleright L_{1}^{\prime} \mid L_{2}$, the well formed configuration $\Gamma_{c} \triangleright L_{1}$ does a reduction to $\Gamma_{c} \triangleright L_{1} \longrightarrow$ $\Gamma_{c} \triangleright L_{1}^{\prime}$ for some h. By induction we know that

a) either $H_{1} \longrightarrow H_{1}^{\prime}$ such that $\phi\left(L_{1}^{\prime}\right)=H_{1}^{\prime}$

b) or $\phi\left(L_{1}^{\prime}\right)=H_{1}$

From the reduction rule reduction rule $(\mathrm{R}-\mathrm{H}-$ CONTX), the $\mathrm{D}_{\pi}$ system $H_{1} \mid H_{2}$ can reduce to $H_{1}^{\prime} \mid H_{2}$. We already know that either $\phi\left(L_{1}^{\prime}\right)=H_{1}^{\prime}$ or $\phi\left(L_{1}^{\prime}\right)=H_{1}$, therefore by definition of $\phi$ we know that, either $\phi\left(L_{1}^{\prime} \mid L_{2}\right) \equiv H_{1}^{\prime} \mid H_{2}$ or $\phi\left(L_{1}^{\prime} \mid L_{2}\right) \equiv$ $H_{1} \mid H_{2}$. The other case in rule (R-CONTX) is exactly similar.

8) let us take second compositional case where a well formed configuration in $\mathrm{DR}_{\pi}^{\varphi}, \Gamma_{c} \triangleright L_{1}$ does a reduction to $\Gamma_{c} \triangleright L_{2}$ using the rule (R-STRUCT)because

$$
\Gamma_{c} \triangleright L_{1}^{\prime} \longrightarrow \Gamma_{c} \triangleright L_{2}^{\prime}
$$

for some $\mathrm{h}$ where $L_{1} \equiv L_{1}^{\prime}$ and $L_{2} \equiv L_{2}^{\prime}$. Let us assume that a $\mathrm{D}_{\pi}$ system $H_{1}$ is such that $\phi\left(L_{1}\right)=H_{1}$. As $L_{1} \equiv L_{1}^{\prime}$ therefore from proposition 2 , we know that $\phi\left(L_{1}\right) \equiv \phi\left(L_{1}^{\prime}\right)$. Now by induction we know that $\Gamma_{c} \triangleright$ $H_{1} \longrightarrow \Gamma_{c} \triangleright H_{2}$ for some $h$ the $\mathrm{D}_{\pi}$ system term $H_{2}$ such that either $\phi\left(L_{2}^{\prime}\right) \equiv H_{2}$ or $\phi\left(L_{2}^{\prime}\right) \equiv H_{1}$. Since it is known that $L_{2} \equiv L_{2}^{\prime}$ and using proposition 2 we know that $\phi\left(L_{2}^{\prime}\right) \equiv \phi\left(L_{2}\right)$ therefore either $\phi\left(L_{2}\right) \equiv H_{2}$ or $\phi\left(L_{2}\right) \equiv H_{1}$.

Theorem 2. In $\mathrm{DR}_{\pi}^{\varphi}$, if a well formed configuration $\Gamma_{c} \triangleright L_{1}$ does a reduction $\Gamma_{c} \triangleright L_{1} \longrightarrow \Gamma_{c} \triangleright L_{2}$ and $\phi\left(L_{1}\right)=H_{1}$ where $H_{1}$ is a $\mathrm{D}_{\pi}$ system if and only if either there exists a $\mathrm{D}_{\pi}$ system $H_{2}$ such that $H_{1} \longrightarrow H_{2}$ and $\phi\left(L_{2}\right) \equiv H_{2}$ or $\phi\left(L_{2}\right) \equiv H_{1}$.

Proof.(OUTLINE) By using lemma.2 and lemma.3.

\section{CONCLUSION}

We described the syntax and reduction semantics for the calculus $\mathrm{DR}_{\pi}^{\varphi}$ that gives a realistic model of distributed network with incorporation of dynamic updation in routing table. We have explained an example to demonstrate reduction rules and also demonstrate that how routing table is updated across the network by using distance vector routing updates. This equivalence has been proved with the well known distributed $\pi$-calculus, $\mathrm{D}_{\pi}$ after abstracting away the unnecessary details from $\mathrm{DR}_{\pi}^{\varphi}$. Now we have proved that both $\mathrm{DR}_{\pi}^{\varphi}$ and $\mathrm{D}_{\pi}$ systems are reduction equivalent after abstracting away the details of routers and paths from $\mathrm{DR}_{\pi}^{\varphi}$.

This calculus implemented routing table updates via distance vector routing methods and included the exclusive feature of this particular calculus with a novel notation $(\longleftrightarrow)$ to depict the exchange of routing tables at global time $t=t_{k^{\prime}}$. Thus all the routing tables are updated dynamically due to this paths are also changed and ensure the best optimal path. This is more close to the actual real distributed network.

In $\mathrm{DR}_{\pi}^{\varphi}$, the $\delta$ function used in the calculus is abstract function which does not allow a real value in the network. Also the calculus does not support dynamic node creation which can be a possible future work for further research. In this paper, we have shown that specification coincides its implementation in our next work will justify the calculus using bisimulation based proof technique.

\section{REFERENCES}

[1] R. Milner., Communicating and mobile systems: The $\pi$-Calculus. Cambridge University Press, 1999.

[2] M. Gaur., "A routing calculus for distributed computing." In Elena Troubitsyna, editor, Proceedings of Doctoral Symposium held in conjunction with Formal Methods 2008,Turku Centre for Computer Science General Publication, vol. 48, pp. 23-32., May 2008.

[3] M. Gaur, "A routing calculus: Towards formalising the cost of computation in a distributed computer network," PhD, Informatics, University of Sussex, U.K., December 2008.

[4] M. Gaur and M. Hennessy, “. counting the cost in the picalculus (extended abstract)." Electronic Notes in Theoretical Computer Science (ENTCS), pp. 229:117-129, 2009.

[5] M. Hennessy., A distributed Pi-Calculus. Cambridge University Press, 2007.

[6] D. Sangiorgi and DavidWalker., The $\pi$-Calculus: A theory of Mobile Processes. Cambridge University Press, 2001. 
[7] J. Parrow., An Introduction to the $\pi$-Calculus, Dep. Teleinformatics,Royal Institue of Technology,Stockholm.

[8] M. Gaur, S. J. Gay, and I. Mackie, "A routing calculus with flooding updates," in Proceedings of the 11th International Conference on Distributed Computing and Internet Technology - Volume 8956, ser. ICDCIT 2015. New York, NY, USA: Springer-Verlag New York, Inc., 2015, pp. 181-186. [Online]. Available: http://dx.doi.org/10.1007/9783-319-14977-6_12

[9] R. Milner, Communicating and mobile systems: The Pi Calculus. Cambridge: Cambridge University Press, 1999.

[10] M. Gaur., "A routing caculus towards formalising the cost of computation in a distributed computer netwok," Ph.D. dissertation, University of Sussex, 2008.

[11] U. Montanari and M. Sammartino, "Network-conscious $\pi$-calculus - A model of pastry," Electr. Notes Theor. Comput. Sci., vol. 312, pp. 3-17, 2015. [Online]. Available: https://doi.org/10.1016/j.entcs.2015.04.002

[12] R. D. Nicola, G. L. Ferrari, and R. Pugliese, "Klaim: a kernel language for agents interaction and mobility," IEEE Transactions on Software Engineering, 1998.

[13] L. Tang and Q. Liu, "A survey on distance vector routing protocols," CoRR, vol. abs/1111.1514, 2011. [Online]. Available: http://arxiv.org/abs/1111.1514

[14] J. J. Garcia-Luna-Aceves, "A unified approach to loop-free routing using distance vectors or link states," SIGCOMM Comput. Commun. Rev., vol. 19, no. 4, pp. 212-223, Aug. 1989. [Online]. Available: http://doi.acm.org/10.1145/75247.75268

[15] M. K. Marina and S. R. Das, "Ad hoc on-demand multipath distance vector routing," SIGMOBILE Mob. Comput. Commun. Rev., vol. 6, no. 3, pp. 92-93, Jun. 2002. [Online]. Available: http://doi.acm.org/10.1145/581291.581305

[16] I. Vukotic and S. Scepanovic, "Description of distance vector routing protocols using mathematical means," 012013.

[17] A. S. Tanenbaum., Computer Networks., 4th ed. Upper Saddle River,New Jersey,: Pearson Education, Inc.,, 2003.

[18] C. Cheng, R. Riley, S. P. R. Kumar, and J. J. Garcia-LunaAceves, "A loop-free extended bellman-ford routing protocol without bouncing effect," in Symposium Proceedings on Communications Architectures \&Amp; Protocols, ser. SIGCOMM '89. New York, NY, USA: ACM, 1989, pp. 224-236. [Online]. Available: http://doi.acm.org/10.1145/75246.75269

[19] D. Walden, "The bellman-ford algorithm and "distributed bellman-ford," 012008

[20] K. R. Hutson, T. L. Schlosser, and D. R. Shier, "On the distributed bellman-ford algorithm and the looping problem," vol. 19, pp. 542551, 112007. 